Service social

\title{
L'histoire du maintien à domicile ou les nouveaux apôtres de l'État
}

\section{Jacques Roy}

Volume 43, numéro 1, 1994

Maintien à domicile

URI : https://id.erudit.org/iderudit/706640ar

DOI : https://doi.org/10.7202/706640ar

Aller au sommaire du numéro

Éditeur(s)

École de service social de l'Université Laval

ISSN

1708-1734 (numérique)

Découvrir la revue

Citer cet article

Roy, J. (1994). L'histoire du maintien à domicile ou les nouveaux apôtres de l'État. Service social, 43(1), 7-32. https://doi.org/10.7202/706640ar
Résumé de l'article

Par cet article l'auteur se propose de retracer les grandes étapes de l'évolution du maintien à domicile au Québec, de l'origine des premiers services offerts par l'État jusqu'à aujourd'hui. Chemin faisant, il compare l'évolution du discours officiel de l'État avec celle de la réalité des services et des clientèles. Il met en évidence quelques contradictions issues de ces deux parcours. $\mathrm{Au}$ terme de l'itinéraire, il s'interroge sur l'avenir du maintien à domicile dans le contexte du désengagement de l'État et de l'affaiblissement des réseaux d'aide naturelle que sont les familles et les communautés. 


\section{A R R T I C L E S}

\section{L'histoire du maintien à domicile ou les nouveaux apôtres de l'État}

Jacques RoY

Sociologue, chercheur au Centre de recherche sur les services communautaires de l'Université Laval et coordonnateur d'une équipe de recherche en soutien à domicile

Retracer les principales phases de l'histoire récente du maintien à domicile, c'est chemin faisant remonter en amont des politiques sociales au Québec. De fait, les services de maintien à domicile offerts par le réseau public - par l'intermédiaire des Centres locaux de services communautaires (CLSC) - se posent comme miroir de l'évolution de la pensée de l'État québécois en matière de politique de santé et de services sociaux. Ils sont la résultante des nombreux changements de cap gouvernementaux, en particulier depuis 1979.

L'objet de cet article consiste donc à mettre en évidence les principaux stades de l'évolution du maintien à domicile en s'attachant de près au contexte qui les a fait naître. Le point de vue qui y est développé est celui d'un ex-coordonnateur de programme qui fut à la barque des services à domicile pendant huit ans au CLSC Des Chenaux et d'un chercheur actuellement rattaché à une équipe de recherche sur le soutien à domicile au Centre de recherche sur les services communautaires à I'Université Laval. Position qui facilitera les emprunts tant au 
milieu des pratiques qu'à celui de la recherche afin de les réunir sous un même chapiteau, le temps d'une histoire.

\section{EN QUÊTE D'UNE DÉFINITION}

Notre propos concerne donc l'itinéraire du maintien à domicile au Québec. Mais qu'est-ce que le maintien à domicile? On aimerait que la réponse soit simple. Elle ne l'est pas! Par exemple, chacun des trois termes suivants - « services», "maintien», "domicile»-constitue un véritable traquenard. Ainsi, les services: il n'en existe pas de liste précise et commune selon les CLSC. Bien sûr, les soins infirmiers sont présents partout, mais certains y ajoutent des services plus spécialisés - telle I'hémodialyse -, d'autres non. Et puis, les services d'entretien ménager font-ils partie de la liste standard des services de l'État? Selon le document Une réforme axée sur le citoyen (MSSS, 1990a), c'est non, mais dans la pratique certains CLSC en offrent, etc. Aucun document gouvernemental n'a pu fournir une liste définitive de services à offrir dans le cadre du maintien à domicile (voir MAS, 1983, 1984 et MSSS, 1988, 1990b, 1991, 1992b et 1993).

Nous venons de dire «maintien » à domicile. Maintient-on les clientèles à domicile ou les soutient-on pour compenser des pertes $d^{\prime}$ autonomie qui, autrement, les confineraient - les maintiendraient à domicile? On aura l'occasion de voir que I'appellation «maintien à domicile » est à l'origine de ces services. Par ailleurs, certains ont modifié l'expression originelle en affirmant que c'est du "soutien à domicile » qui est visé par les activités visant l'autonomie de la personne dans son milieu naturel. Mais d'autres, prenant acte d'un alourdissement considérable des clientèles, évoquent aujourd'hui qu'on "maintient» désormais à domicile ces usagers qui ont peu de chance de voir restaurer leur autonomie. Alors, en CLSC, on «maintient» et on "soutient» à domicile. D'où l'appellation non contrôlée, qui varie d'un CLSC à l'autre.

Bon! Mais le domicile de l'usager, c'est tout de même simple, évident. La maison, c'est du domicile, I'hôpital, non. Pas plus que le centre d'accueil public ou privé conventionné. Mais d'autres catégories sont encore sujettes à interprétation. Les résidences pour personnes âgées avec permis, sans permis, les conciergeries spécialisées avec services, sans services, les chambres et pensions, les foyers illicites, les HLM... Certains CLSC s'y rendent, d'autres non. La zone est obscure. Tout est discutable.

Ce bref détour concernant ces premières notions de base illustre le caractère éclaté, non réductible du maintien à domicile. C'est le fil 
d'Ariane que nous suivrons tout au long des différents stades du maintien à domicile. Pour les fins de présentation, on retiendra quatre stades qui caractérisent l'évolution du maintien à domicile parallèlement à celle des politiques sociales au Québec.

\section{Premier Stade: Du credo de l'institutionnalisation à l'idéologie du "domicile»}

Sous une forme ou sous une autre, les services de maintien à domicile ont toujours existé. Ainsi, en 1962, des unités de soins à domicile offraient des services à une clientèle, qui visaient à éviter chez elle les hospitalisations précoces et à favoriser un prompt retour à domicile des personnes hospitalisées. Ces unités de soins seront intégrées au réseau public de services avec la Loi sur les services de santé et les services sociaux (loi 65) (chap. 48, 1971). Aux soins infirmiers s'ajouteront progressivement les services d'aide à domicile afin d'assister dans leur quotidien des personnes en perte d'autonomie (MSSS, 1988, 1991 et 1992b).

C'est véritablement dans les années 70 que l'État québécois a choisi de structurer ces services et de les prendre en charge. Dès 1977, le gouvernement fédéral facilite le développement de tels services en subventionnant les provinces désireuses de rendre universel l'accès aux services à domicile 1 . Au Québec, les départements de santé communautaire devaient fournir les soins à domicile, alors que les Centres de services sociaux couvraient les services d'aide à domicile (entretien ménager, préparation de repas, etc.). Progressivement, au tournant des années 80 , le réseau des CLSC devait se voir transférer la totalité des services de maintien à domicile pour en assumer l'entière responsabilité (MSSS, 1993).

Dans les années 70, l'État québécois inscrit ses visées: il désire progressivement offrir aux personnes âgées, en plus d'une politique globale du vieillissement, des services favorisant le maintien à domicile qui auraient pour effet de fournir une solution de remplacement à I'institutionnalisation pour ces personnes en perte d'autonomie (Bolduc, 1988; Grandmaison, 1982). Voilà une première obsession de l'État: la spirale montante de l'institutionnalisation. Du reste, lui-même y avait largement contribué en assurant la mise en place de services gérontologiques calqués à partir d'une philosophie « anti-âgiste » (selon le concept de Zay, 1984) où l'État se faisait protecteur des personnes âgées en privilégiant l'hébergement en centre $d^{\prime}$ accueil comme réponse aux

1. Voir I'article de Lesemann et Nahmiash: «Canada, logiques hospitalières et pratiques familiales de soins (Québec)», dans Lesemann et Martin (1993). 
besoins des aînés en perte d'autonomie. À cette époque (années 60, 70), on construisait partout au Québec des centres d'accueil au rythme des polyvalentes. " [...] c'était la période de I'hôtellerie où I'on offrait à une partie de la population une chambre d'hôtel comme récompense pour services rendus à la nation» (Guberman et al., 1987).

Ambivalence de l'État, schizophrénie dans le discours, toujours est-il que l'essentiel des ressources publiques est consacré à l'hébergement institutionnel des personnes âgées. Avec pour résultat qu'au début des années 80 le Québec enregistrait l'un des taux d'hébergement en institution des aînés les plus élevés en Occident. Avec $7 \%$ à $8 \%$ des personnes de 65 ans et plus vivant en institution au début des années 80 et selon les plus récentes estimations (Trahan, 1989), le Québec supplante des pays tels I'Australie, la France, I'Angleterre, les États-Unis, I'Autriche, la Suède ou les Pays-Bas, dont les taux s'échelonnaient de $3,5 \%$ à 6,3\% pour des périodes comparables (Brink, 1985).

Nous parlons de personnes âgées, car l'histoire du maintien à domicile est intimement liée à elles. Les personnes de 65 ans et plus représentent de fait la grande majorité de la clientèle des services de maintien à domicile, soit environ $70 \%$ (MSSS, 1991). De plus, elles constituent l'une des catégories de population les plus visées par les objectifs de désinstitutionnalisation proposés par les politiques sociales de I'État québécois en matière de santé et de services sociaux au tournant des années 80 . Mais d'autres groupes, dès I'origine des services, étaient également visés: les personnes handicapées (par le truchement de l'OPHQ), les malades chroniques, les personnes post-hospitalisées, les familles en difficulté. Suivront quelques années plus tard la clientèle en phase terminale, les cas de santé mentale et, plus récemment, les personnes victimes du sida.

C'est en 1979 que l'État québécois se dote d'une politique officielle en matière de services à domicile ${ }^{2}$. Le document précise sa définition et les objectifs poursuivis :

Par services à domicile, on entend l'ensemble des activités visant à apporter au domicile du bénéficiaire un soutien moral, matériel et médical susceptible de compenser une perte d'autonomie, de prévenir ou de pallier à une situation de crise et d'éviter ou d'abréger le séjour en institution ${ }^{3}$.

2. Bien qu'une première version ait été produite en 1976, on fait toujours référence au document de 1979 comme étant la première position officielle du Ministère.

3. Ministère des Affaires sociales, Les services à domicile. Politique du ministère des Affaires sociales, Québec: Gouvernement du Québec, 1979, p. 7. 
L'intention gouvernementale était la suivante: effectuer un virage à cent quatre-vingts degrés sur le plan des politiques à l'égard des personnes en perte d'autonomie à domicile (principalement des personnes âgées) en leur offrant un réseau complet de services à domicile se posant comme solution de remplacement à I'hébergement institutionnel. Des motivations certaines appuyaient cette volonté de réorientation politique; trois d'entre elles méritent d'être soulignées. En premier lieu, l'État prend acte d'un courant de pensée de plus en plus dominant vers la fin des années 70 qui visait la prise en charge par les personnes de leur propre santé et la volonté de s'affranchir de la dépendance professionnelle des services de l'État-providence. Relativement à cette "dépendance », on soulignait notamment le caractère «abrutissant » du professionnalisme dans les services (McKnight, 1977). Panet-Raymond (1987) évoque que la nature bureaucratique et paternalisante de l'approche institutionnelle et professionnelle suscitait des remises en question fondamentales. Sorte de retour de I'histoire donc, où la famille et les ressources communautaires étaient auparavant honnies ou ignorées et les services professionnels, adulés : les personnes âgées «méritaient » plus que la famille pour les soutenir à domicile et l'hébergement institutionnel faisait figure d'Eldorado sur le plan des services. La politique de 1979 opère une première rupture importante avec cette vision des services ${ }^{4}$. À cette époque, l'État pouvait alors soutenir ce discours promaintien à domicile puisqu'il couvrait à la fin de la décennie 70 une clientèle en légère perte $d^{\prime}$ autonomie qui avait besoin d'à peine quelques heures de services par semaine (ou par mois), du lundi au vendredi (Bolduc, 1988). Les autres clientèles, plus lourdes, prenaient la direction de l'hébergement institutionnel.

Mais, progressivement, se profilait une clientèle en plus grande perte d'autonomie à l'intérieur des services de maintien à domicile. Celle-ci se situait à la frontière de l'hébergement institutionnel. Afin de répondre aux besoins grandissants de cette nouvelle clientèle, l'État devait, parallèlement aux services à domicile, créer de nouvelles ressources, telles que les centres de jour, les hôpitaux de jour, les unités psycho-gériatriques par exemple; un peu plus tard (1986) s'ajouteront une nouvelle génération de services à domicile, soit les services intensifs de maintien à domicile (SIMAD) (nous en discuterons plus loin). Selon le postulat de la politique des services à domicile de 1979 (MAS, 1979), ces services devaient diminuer le recours à l'hébergement institutionnel (figure de proue de la professionnalisation des services et de la

4. D'autant plus que, selon l'étude de Sicotte (1982), la moitié de la clientèle en centre $\mathrm{d}^{\prime}$ accueil d'hébergement (46\%) aurait pu vivre à domicile avec des services de soutien appropriés (Sicotte, 1982, p. 18). 
dépendance). Des chercheurs ont alors entrepris de vérifier l'impact des services de maintien à domicile sur le désir d'hébergement institutionnel de la clientèle à domicile (Béland, 1982, 1983, 1984 et 1987) ou sur le profil d'utilisation des ressources socio-sanitaires (Contandriopoulos, 1983 et 1984). Leur verdict fut sans appel : I'impact des services à domicile était à peu près nul. La clientèle des services à domicile désirait tout autant être hébergée en institution et elle consommait autant de services médicaux, hospitaliers et autres dans le réseau de la santé.

D'autres auteurs ont par ailleurs remis en question la pertinence de telles évaluations dans un contexte où les services à domicile offerts étaient de courte durée et de faible intensité, donc n'avaient pas les propriétés nécessaires pour répondre aux besoins de cette clientèle en perte sérieuse d'autonomie (Bolduc, 1986; Roy, 1989, 1990b).

Le second motif associé au virage de l'État vers le maintien à domicile est relié à la perspective du vieillissement accéléré de la population et des conséquences de ce vieillissement sur les services. Considéré comme l'une des plus jeunes sociétés en Occident à la fin des années 70 et au début des années 80, le Québec connaîtra l'un des vieillissements les plus rapides et deviendra dans quarante ans l'une des deux ou trois populations les plus vieilles au monde (Mathews, 1988b). À titre d'illustration, mentionnons que le groupe des personnes âgées de 75 ans et plus, soit le groupe de loin le plus dépendant des services de l'État et celui qui pèse le plus lourd en matière de financement des services de santé (Dossier "personnes âgées», Commission Rochon, 1987; Mathews, 1988a), s'accroît chaque année dix fois plus rapidement que le reste de la population; ainsi, selon les prévisions du Bureau de la statistique du Québec, les 75 ans et plus enregistreront un bond de 102,3\% entre 1981 et I'an 2001, alors que la population du Québec marquera une hausse de $9,7 \%$ pour la même période (BSQ, 1984).

Une telle perspective d'explosion démographique des aînés motive donc l'État à réviser sa politique d'institutionnalisation des personnes âgées en perte d'autonomie, les termes de celle-ci conduisant tout droit à I'utopie dans I'avenir. Cela nous introduit tout naturellement au dernier motif principal: les contingences de l'État sur le plan des finances publiques.

Indépendamment de l'évolution démographique en spirale des personnes âgées, intervient au début des années 80 la crise économique qui fait ravaler à l'État même ses aspirations les plus modestes en matière de politique de vieillissement et de services de santé. La «cassure » du budget gouvernemental se présente au moment même où I'État désire effectuer une réorientation vers le maintien à domicile. Deux phénomènes se produisent: pas d'argent neuf pour la mise en 
place de nouveaux services et les seules disponibilités budgétaires serviront à répondre à l'alourdissement de la clientèle en hébergement institutionnel (I'État doit alors assumer la responsabilité de ces choix de politiques antérieurs).

Discours de maintien à domicile, budget vers l'hébergement institutionnel, voilà le portrait au début des années 80 : un virage politique sur papier!

Pour Therrien (1989), les conséquences sur les services de maintien à domicile seront majeures :

Les restrictions budgétaires ont mené à un rationnement des ressources tel que les mesures envisagées n'ont été réalisées qu'à moitié. La volonté de limiter les places en institution a produit un effet en cascade qui a mené à l'engorgement des autres services par des cas trop lourds pour leurs ressources, les empêchant de se consacrer à la réalisation de leurs propres objectifs $[\ldots]$. Cette situation se répercute doublement sur les services à domicile conçus au départ comme la pièce maîtresse du maintien à domicile: tout en obtenant une portion congrue des budgets, on cherche à les orienter vers des cas plus lourds (Therrien, 1989, p. 158).

Ouvrons une parenthèse: la tentation est forte ici d'établir un parallèle entre les politiques de services à l'égard des personnes âgées et le champ de la santé mentale. Dans les deux cas, on a désinstitutionnalisé sans pour autant mettre en place les ressources nécessaires pour répondre aux besoins des clientèles dans leur milieu naturel et au sein de leur communauté (Bolduc, 1986; Comité de la santé mentale, 1987). Une exception: les personnes handicapées. Cette clientèle jouira d'un statut particulier, bénéficiant de plans de services à domicile gérés à I'origine par I'Office des personnes handicapées du Québec (OPHQ, 1984). En 1984, le fonds pour les personnes handicapées sera régionalisé et administré par les CRSSS (Bolduc, 1988) et c'est en 1989 que le réseau de la santé et des services sociaux se verra accorder la responsabilité entière des services de maintien à domicile pour les personnes handicapées (MSSS, 1990b). C'est d'ailleurs à partir de la problématique des personnes handicapées qu'on a expérimenté la formule dite $\mathrm{d}^{\prime}$ " «allocation directe ${ }^{5}$ » en I'extensionnant aux autres clientèles du maintien à domicile (Bolduc, 1981a, 1988; MSSS, 1990b).

5. Par cette formule, on établit le besoin de services d'aide à domicile de l'usager et l'on remet une allocation financière à ce dernier pour qu'il s'achète lui-même des services. Pour l'année 1988-1989, 65 \% des CLSC au Québec avaient recours à cette formule pour l'ensemble de la clientèle du maintien à domicile. Globalement, la formule s'adressait à $15 \%$ de la clientèle et elle totalisait $17 \%$ du budget en maintien à domicile (MSSS, 1990b). 
Donc, le courant de déprofessionnalisation, le "géronto-boom» démographique et un État "cassé » sur le plan des finances publiques par la crise économique du début des années 80 incitent principalement l'État à réorienter ses politiques en «faveur » du maintien à domicile. Parallèlement, une nouvelle perspective se dessine: I'alourdissement des clientèles, tant en maintien à domicile qu'en hébergement institutionnel.

\section{DEUXIÈME STADE: L'alourdissement des clientèles ou la fin du tender loving care}

À l'origine, les services de maintien à domicile s'adressaient à une clientèle en légère perte d'autonomie. Rapidement, à la faveur de la politique de désinstitutionnalisation (notamment dans les secteurs de la santé mentale [Comité de la santé mentale, 1987], des personnes handicapées [OPHQ, 1984] et des aînés(es) [MAS, 1985]), les clientèles devaient se métamorphoser rapidement en cas de plus en plus lourds pour les services. "Bien fini le temps des "brunches" et du tender loving care, qui ont marqué les débuts des CLSC il y a 15 ans. En miettes, le mythe des services "chromés" ", rappelait Huguette Roberge dans La Presse du 4 octobre 1987 pour illustrer la transformation radicale qu'ont dû opérer les services de maintien à domicile pour répondre aux besoins d'un véritable "tiers monde montant » chez la clientèle.

Trait historique: le regard porté sur les pratiques et la clientèle en maintien à domicile du côté gouvernemental en particulier a toujours accusé un retard sur la réalité. Il en est ainsi du regard porté sur le phénomène $d^{\prime}$ alourdissement de la clientèle en maintien à domicile. Le ministère des Affaires sociales, qui avait la charge de défendre des crédits additionnels auprès du Conseil du trésor afin d'appuyer sa nouvelle politique de services à domicile, conservait encore, même au milieu des années 80 , l'image de clientèles en légère perte d'autonomie en maintien à domicile, ne nécessitant que des services de faible intensité et de façon irrégulière. On verra plus loin que la réalisation de monographies tirées de l'observation de clientèles en maintien à domicile, effectuée par la Direction des services communautaires du Ministère en 1986, transformera radicalement cette perception. Pour le moment, retenons deux facteurs principaux (outre la crise des finances publiques du début des années 80 , les engagements à l'égard de la clientèle en centres d'accueil d'hébergement et la méconnaissance du Ministère relativement au phénomène montant de l'alourdissement) qui ont nui considérablement au Ministère pour négocier des enveloppes de développement en maintien à domicile auprès du Conseil du trésor (et retarder ainsi l'implantation d'une véritable politique de maintien à domicile). 
Premier facteur: Le Ministère se tire lui-même dans le pied. De fait, chaque fois qu'il se présente au Conseil du trésor, celui-ci lui renvoie la conclusion d'une étude menée au Ministère (Béland, 1982) selon laquelle les services de maintien à domicile $n^{\prime}$ ont pas d'impact sur les désirs d'hébergement institutionnel des personnes âgées. Comment, pour le Conseil du trésor, entrevoir alors des économies en investissant davantage pour des services à domicile qui ne réduiraient pas l'achalandage au palier de l'hébergement institutionnel? C'était pourtant un objectif explicite de la politique des services à domicile (MAS, 1979). Et quelle aubaine de détenir une copie d'une telle étude effectuée par le ministère demandeur!

Second facteur: L'inexistence d'informations sur le maintien à domicile. Quelles que soient les questions posées aux fins de financement du programme de maintien à domicile (combien de personnes sont couvertes, pour combien d'heures de services par semaine et pour quels types de services? À quelle catégorie de clientèles appartiennent-elles? Quelle est la durée moyenne des services reçus ?...), aucune information n'était disponible, du moins jusqu'en 1986. Le Conseil du trésor n'aurait même pas eu le loisir d'utiliser le sarcasme de St-Exupéry voulant que les chiffres cachent plus de choses qu'ils n'en révèlent: il n'y avait pas de chiffres. Voilà qui handicape singulièrement toute demande de crédits supplémentaires au Ministère où la vertu même du maintien à domicile n'a pu à elle seule émouvoir les analystes du Conseil. Résultat pour la première moitié des années 80 : des services compromis, eu égard aux nouveaux besoins des clientèles, par un sous-financement chronique. Parallèlement, le réseau des établissements d'hébergement institutionnel réussissait à obtenir des fonds de développement en se basant sur un système d'information sur les clientèles et les services. Ses demandes étaient appuyées par des profils d'information relativement complets, mettant en perspective l'écart entre les besoins des clientèles et le niveau de ressources existant.

1986 constitue une année-charnière dans ce second stade de l'évolution du maintien à domicile. Trois événements viendront marquer la reconnaissance du phénomène d'alourdissement des clientèles (tant sur le plan descriptif qu'en matière d'action gouvernementale) et l'émergence d'une nouvelle génération de services de maintien à domicile. D'abord, un premier bilan du maintien à domicile au Québec est dressé par la Fédération des CLSC du Québec (Roy, 1986). Ensuite, ce bilan est suivi de neuf monographies produites par le Ministère, décrivant la réalité des clientèles et des services à domicile (Filiatrault et Locas, rédigées en 1986 et publiées en 1987). Enfin, la ministre des Affaires sociales de l'époque, madame Thérèse Lavoie-Roux, met sur pied à I'automne 1986 un train de mesures visant le désengorgement des salles 
d'urgence en milieu hospitalier; parmi ces mesures, on assiste à l'implantation de services intensifs de maintien à domicile (SIMAD), qui marqueront une rupture capitale avec la première génération des services réguliers de maintien à domicile. Regardons chacun de ces événements en ce qu'ils nous parlent de l'évolution du maintien à domicile.

Au printemps 1986, la Fédération des CLSC du Québec publie un bilan du maintien à domicile réalisé auprès de 106 CLSC. Ce bilan trace le portrait des clientèles, des services et des ressources affectées à ce programme. Les faits saillants du bilan ont permis une présentationchoc dans le cadre d'un colloque sur le maintien à domicile en mars 1986. Retenons les plus marquants:

- L'alourdissement des clientèles et l'insuffisance de ressources en maintien à domicile sont considérés comme les deux problèmes prioritaires par les CLSC.

- Parmi les personnes âgées, 12 \% sont couvertes par les services de maintien à domicile, soit deux fois moins que la proportion d'aînés requérant de tels services.

- Afin de répondre aux besoins grandissants des clientèles, sans ressources additionnelles, les CLSC ont mis en œuvre deux modèles de pratique. L'un vise à accorder moins d'heures de services par usager afin d'offrir une plus grande pénétration de clientèle. L'autre fait l'inverse: moins d'usagers, plus de services par usager.

- Les trois quarts des CLSC (73\%) accusaient en 1984-1985 un déficit sur le programme de maintien à domicile.

- Il faudrait quadrupler les ressources en maintien à domicile pour répondre aux besoins des clientèles qui s'alourdissent et se multiplient sans cesse (Roy, 1986).

Quadrupler le budget du maintien à domicile équivalait en 1986 à le porter de 80 millions à 320 millions. À un moment où aucun gouvernement occidental n'aurait pu s'aventurer dans de telles dépenses. Malgré tout, cette évaluation (multiplier par quatre les ressources en maintien à domicile) fut reprise et accréditée par les travaux de la Commission Rochon portant sur le dossier des personnes âgées (Commission Rochon, 1987).

Le bilan avait un premier mérite: celui de faire exister le maintien à domicile en chiffres. Pour le reste, il dressait un portrait du maintien à domicile, bien connu du milieu des pratiques mais largement inconnu du grand public comme du ministère des Affaires sociales à l'époque. Devant un tel constat (alourdissement des clientèles, insuffisance de ressources...), le Ministère voulut en avoir le coeur net; il procéda à la 
réalisation de neuf monographies sur le maintien à domicile en autant de CLSC (Filiatrault et Locas, 1987). Les conclusions du bilan ébranlaient sa propre représentation du maintien à domicile.

La synthèse de ces monographies «marque l'ébahissement des observateurs, proprement tombés des nues devant la lourdeur des cas traités par les équipes de maintien à domicile des CLSC, la sousbudgétisation de ce programme... et la qualité des services dispensés par ces mêmes équipes, eu égard à leurs moyens dramatiquement insuffisants» (Roberge, 1987). La prise de conscience se fixe, un consensus se tisse: il faudra consolider le maintien à domicile dans la perspective de nouvelles clientèles en perte sérieuse d'autonomie.

À l'automne 1986, la ministre Lavoie-Roux donne un véritable coup de barre pour redresser la situation des urgences en milieu hospitalier. Parmi les différentes mesures adoptées, on choisit d'implanter un programme de services intensifs de maintien à domicile (SIMAD). Les villes de Montréal, Québec et Trois-Rivières ont été retenues comme lieux d'expérimentation ${ }^{6}$.

Ces services intensifs de maintien à domicile visaient en premier lieu à contribuer au désengorgement des salles d'urgence des hôpitaux de courte durée. Chemin faisant, on leur ajoutera deux autres objectifs, soit favoriser le maintien à domicile des personnes âgées en perte $d^{\prime}$ autonomie et offrir une ressource de remplacement à l'institutionnalisation des aînés en perte d'autonomie (Joubert, Laberge, Fortin, Paradis, Desbiens, 1991). Quittant le corridor des une à trois heures de services par semaine, par usager (contexte des services réguliers), les services SIMAD pouvaient offrir en moyenne une vingtaine $d^{\prime}$ 'heures de services par semaine, par usager (Joubert et al., 1991). Les clientèles de ces services se regroupaient dans les catégories suivantes, selon le motif d'admission aux services:

$\begin{array}{lr}\text { Phase préterminale ou terminale } & 25 \% \\ \text { Attente d'hébergement } & 9 \% \\ \text { Post-hospitalisation } & 13 \% \\ \text { Maintien à domicile à long terme } & 53 \%\end{array}$

Note: Près du quart de la clientèle (23\%) était affectée par des troubles neuropsychiatriques.

Source: Joubert et al., 1991, p. 57.

Pour révolutionnaires qu'ils aient été à cette époque dans la tradition des services de maintien à domicile existants (de faible intensité et de

6. Plus précisément, les régions administratives 03 (Québec), 04 (Trois-Rivières), 06A (Montréal-Métro), 06B (Laurentides-Lanaudière) et 06C (Montérégie). 
courte durée), les services SIMAD puisaient leur origine dans des expériences déjà connues et datant de quelques années ${ }^{7}$. En particulier, leur conception a été inspirée de l'expérience du projet intitulé "Cas lourds » administré par le CRSSS du Montréal métropolitain à compter de 1981. L'objectif de ce projet consistait en la couverture de clientèles trop lourdes pour les services réguliers; les personnes en attente d'hébergement institutionnel, en phase terminale, en situation de crise ou en maintien à domicile à long terme figuraient parmi la clientèle de ces services (CRSSSMM, 1985 ; Lambert et Drabble, 1985 ; Trépanier, 1985 et 1986 ; Trépanier et Lambert, 1987). Le succès de cette formule fut reconnu par certains auteurs (notamment Hajjar et Lefebvre, 1988); I'expérience de Montréal servit donc de référence pour l'implantation des services SIMAD. À I'instar des services offerts dans le cadre du projet "Cas lourds» à Montréal, les services SIMAD devaient présenter des résultats positifs, notamment sur le plan de la diminution du recours aux ressources hospitalières et d'hébergement institutionnel (Joubert et al., 1991).

Le phénomène d'alourdissement accéléré des clientèles des services de maintien à domicile $\mathrm{n}^{\prime}$ allait pas sans amener ces derniers à s'interroger sur leur façon de faire. Coincés dans une impasse probante dont ils ne se sont pas encore remis - le maintien à domicile qui représentait moins de $2 \%$ de l'ensemble des dépenses de santé au Québec en 1984-1985 (Roy, 1986) accueillait malgré tout, tel un déversoir universel, toutes les clientèles refoulées aux paliers institutionnels du réseau des affaires sociales -, les CLSC, comme nous I'avons déjà souligné, ont adopté en réaction deux modèles de pratique: plus d'usagers, moins de services pour chacun/plus de services par usager, moins d'usagers. Mais, à l'étage supérieur de la prestation de services - ministère des Affaires sociales, Fédération des CLSC du Québec, autres instances ministérielles et du réseau -, on commençait à jongler avec I'idée de transgresser la règle de l'universalité des services pour dénouer I'impasse. Fruit des politiques sociales d'avant les années 80, les contingences financières de l'État forçaient désormais les décideurs à distribuer autrement les services en ciblant cette fois-ci les populations à atteindre. Le rapport Brunet sur l'avenir des CLSC (Brunet et al., 1987) pratiqua une première brèche: les CLSC devaient maintenant s'occuper en priorité des clientèles desservies par un tronc commun de programmes (six en tout, dont un au choix du CLSC). Fini le caractère tous azimuts des services en CLSC.

7. Nous avons à l'esprit deux expériences. La première, menée dans la région de Québec auprès de personnes handicapées physiques, était liée à la problématique du logement et des services de soutien (Bolduc, 1981b). La seconde expérience portait sur le projet "Cas lourds » de la région de Montréal (CRSSSMM, 1985). 
Prenant acte de ce virage, un mémoire de la Fédération des CLSC du Québec vint préciser les paramètres et balises souhaités pour le programme de maintien à domicile (FCLSC, 1988). L'entreprise de recentrage trouva sa confirmation officielle dans Une réforme axée sur le citoyen (MSSS, 1990a). Paramètres et balises - du moins dans leur acceptation générale - étaient tracés.

Pendant toute cette période, la spirale de l'alourdissement des clientèles poursuivait sa trajectoire. Les intervenants - tels des apôtres devant la traversée du désert, mais sans Dieu croyant à leur mission $\mathrm{n}^{\prime}$ en croyaient pas leurs yeux. Des cas impossibles - grands malades chroniques, personnes lourdement handicapées, personnes âgées présentant des problèmes neuropsychiatriques, mourants en phase terminale - s'adressaient à eux par l'entremise de leur famille pour qu'ils les soutiennent à domicile, alors que le coffre des services à domicile était à sec. Alors aussi que les institutions d'hébergement et les établissements hospitaliers affichaient fermés pour cause de listes d'attente plus longues que celles de la DPJ. Que faire, sinon trouver de nouvelles alliances: les familles, la communauté. Forces réelles ou utopies célestes, toujours est-il que les familles et les communautés s'avéraient l'issue de secours désignée pour partager les termes de l'avenir. D'autant plus que l'on assistait à un curieux renversement des perspectives: I'hébergement institutionnel se posait progressivement comme solution de remplacement au maintien à domicile, alors que ce dernier avait été conçu précisément pour être la solution de remplacement à l'institution (Roy, Vézina et Paradis, 1992). Voilà les intervenants lancés dans une nouvelle évangélisation des ressources du milieu "naturel», afin de compter sur de nouveaux partenaires prêts à les épauler, disposés dans certains cas à se rendre aux limites du burn-out pour répondre aux besoins des clientèles à domicile. Clientèles, répétons-le, principalement issues du courant de désinstitutionnalisation traversant les politiques sociales de l'État au tournant des années 80.

\section{TROISIÈME STADE: La famille et la communauté au secours de l'État}

Recibler les clientèles et sabrer progressivement dans I'universalité des services ne pouvaient suffire à relever les défis qui se posaient à l'État. Il fallait davantage construire un discours autour de la valorisation des forces sociales, des solidarités naturelles, de la famille, des proches, des groupes communautaires qui viendront au secours de l'État. Et pour cause: de $70 \%$ à $80 \%$ de l'aide fournie aux personnes âgées proviendrait d'eux (principalement la famille) (Commission Rochon, 1987). Le document de politique à l'égard des personnes âgées du ministère des 
Affaires sociales (MAS, 1985) donnait le coup d'envoi. Les familles et les communautés étaient élevées au rang de premier acteur de la santé et du bien-être des aînés. C'est aussi l'éloge du partenariat. Tous les documents d'orientation qui ont paru par la suite devaient s'inscrire dans cette perspective commune (Comité de la santé mentale, 1987; Commission Rochon, 1988; MSSS, 1989a et b, 1990a, 1992a; Pelletier et al., 1991).

Dans le cas du maintien à domicile, la nouvelle perspective ouvrit le champ à de multiples expériences associant milieu naturel et intervenants auprès des clientèles en perte d'autonomie à domicile. L'une des plus connues et des plus structurées est sans doute celle découlant de l'approche communautaire telle qu'élaborée par Guay et al. (1985) et mise en application par le Bureau de recherche en intervention communautaire (BRIC) dans bon nombre de CLSC.

Notre propos ne consiste pas à évaluer la portée ou l'intérêt de ces avenues visant à conscrire familles et communautés comme partenaires de l'État en maintien à domicile. Nous rendons compte de leur existence et du contexte général des politiques sociales dans lesquelles elles s'inscrivent. Notons par ailleurs que le rapprochement État-famillecommunauté, sous I'angle des rapports engagés entre eux, a donné lieu à différentes réflexions critiques dans la littérature récente. Devant l'enthousiasme du nouveau discours gouvernemental, certains auteurs ont évoqué le piège $d^{\prime}$ une "survalorisation indiscriminée des systèmes de support naturel »(Corin et al., 1984), alors que d'autres prenaient acte du désengagement de l'État (Therrien, 1989). Plusieurs s'inquiétaient du sort des femmes comme aidantes naturelles prenant le relais de I'État (Fortin, 1987; Guberman, 1987; Jutras et Veilleux, 1989; Therrien, 1989). Nancy Guberman résume ainsi la question:

Quand le livre vert (Pour les familles québécoises, 1984) indique que l'État doit cesser de se substituer à d'autres réseaux d'aide, à des réseaux dits "naturels », il faut constater que, malheureusement, les appels «à la nature des choses» ont rarement bénéficié aux femmes; plus souvent qu'autrement, ils ont servi à confiner les femmes dans la sphère domestique. Si l'État se désengage de l'aide aux personnes, quels sont, en fait, les aidants «naturels » qui s'y substitueraient? Qui d'autre que les femmes ${ }^{8}$ ?

Au-delà de leur volonté de prendre en charge leurs membres en perte d'autonomie à domicile, les familles et les communautés en

8. Nancy Guberman, "Discours de responsabilisation de la "famille" et retrait de l'Étatprovidence ", Couples et parents des années quatre-vingt, Québec: Institut québécois de recherche sur la culture, 1987, p. 200. 
ont-elles les capacités (et aussi le goût)? Sont-elles à la mesure du discours officiel, de la carrure de ses épaules? Certains en doutent (Fortin, 1987 ; Roy, 1988 ; Therrien, 1989). Pour leur part, Jutras et Veilleux posent le dilemme suivant:

Les économies collectives réalisées par la non-institutionnalisation ne doivent pas se traduire par I'appauvrissement des familles et la dégradation du niveau de santé de l'ensemble de la population ${ }^{9}$.

Mais l'État, tête baissée, fonce vers le partenariat. Un document du Conseil de la famille révélait qu'au Québec «Le partenariat est chose acquise, en théorie du moins, en ce qui concerne les décisions importantes » (Conseil de la famille, 1989). Peu importe si la notion de partenariat, telle qu'elle est utilisée dans le discours officiel, se révèle, selon Jutras et Veilleux (1989), embryonnaire, peu opérationnelle et inspirée par une conception pragmatique plutôt qu'idéologique. Peu importe également si les postulats du discours officiel reposent sur des prémisses théoriques plutôt qu'empiriques (vieux travers latin de l'État québécois, qui le distingue à l'occasion du monde anglo-saxon en ce qu'il décrète des politiques et des orientations sans expérimentations préalables et en faisant table rase du passé).

Qu'à cela ne tienne, I'État québécois est bel et bien engagé dans la voie "partenariale». Un document ministériel, datant de 1989, résume bien ce qu'on entend par la notion de partenariat du côté gouvernemental :

Le partenariat suppose la mobilisation de la personne, de ses proches, des intervenants, de la communauté, des ressources publiques et de celles du milieu. Il implique trois conditions : la reconnaissance du potentiel de chacun des partenaires, I'existence de rapports ouverts entre ceux-ci et l'adoption d'objectifs communs $^{10}$.

Si, à l'origine, la mise en œuvre de politiques visant le partenariat n'était pas fondée sur une connaissance approfondie des différents acteurs, de leurs rôles et de leurs limites, il n'en demeure pas moins que des efforts sont présentement consentis dans cette direction par le MSSS. Un exemple éloquent: I'étude sur l'aide par les proches menée par Bolduc et Garant (1990) de la Direction de l'évaluation du Ministère.

9. Sylvie Jutras, France Veilleux, avec la collaboration de Marc Renaud, Des "partenaires » méconnus: Les aidants des personnes âgées en perte d'autonomie, Montréal: Université de Montréal, 1989, p. 55.

10. Ministère de la Santé et des Services sociaux, Politique de santé mentale, Québec: Gouvernement du Québec, 1989, p. 26. 
Ce détour sur les politiques de type "partenariat» de l'État québécois n'est pas inutile pour notre propos - I'histoire du maintien à domicile -, car ce dernier est nettement conditionné par ces nouvelles orientations. Dans le contexte du maintien à domicile, le partenariat va remettre en question les dynamiques existant entre les usagers des services, leur famille et l'État (par l'intermédiaire des services de maintien à domicile qu'il offre). Deux études québécoises s'y sont entre autres attardées dans le champ du maintien à domicile.

Dans leur étude portant sur des personnes en perte d'autonomie à domicile et bénéficiant des services de maintien à domicile de l'État, Lesemann et Chaume (1989) mettent en évidence l'existence de deux logiques parallèles, I'une, familiale, tenant essentiellement à la solidarité, à l'affectif, à la réciprocité et au don entre les membres de la famille et l'autre, étatique (les auteurs parleront «d'économie institutionnelle »), s'appuyant sur le système de production de services de l'État, visant des objectifs abstraits d'universalité et d'équité, dépersonnalisés et en marge du milieu. Deux logiques parfois divergentes mais toujours différentes, qui coexisteraient à l'intérieur d'un partenariat en maintien à domicile. Les auteurs soulignent que les familles ne pensent pas "État», qu'elles ne souhaitent pas devenir un "partenaire » avec lui dans la perspective d'une coproduction de services. Tout en appréciant I'appui des services de maintien à domicile offerts par l'État, les familles, selon la recherche, désirent être autonomes et être les premières responsables des décisions ultimes visant le maintien à domicile ou I'hébergement institutionnel d'un parent âgé (94\% des aidants familiaux partageaient cette opinion dans l'enquête).

Pour leur part, Roy, Vézina et Paradis (1992) ont examiné les dynamiques relationnelles existant entre des bénéficiaires en sérieuse perte d'autonomie, recevant des services SIMAD, leurs aidants familiaux et les intervenants des CLSC fournissant les services SIMAD. Cette recherche conclut à: 1) l'engagement profond des aidants naturels qui soutiennent un aîné en sérieuse perte $\mathrm{d}^{\prime}$ autonomie à domicile - même si l'on ne peut assimiler cet engagement à celui des familles puisque, dans la majorité des cas, l'aide provenait d'un seul membre de la famille (généralement une femme) -; 2) la volonté affirmée des bénéficiaires de demeurer à domicile jusqu' aux limites du possible - l'existence d'une culture anti-hébergement institutionnel a été mise en évidence -; 3) l'insuffisance du volume de services offerts par l'État en regard de leurs conditions nettement détériorées; enfin, l'importance de services tels que le répit ou l'entretien ménager. Ce dernier service nous permet d'ouvrir une brève parenthèse: alors qu'il existe un consensus tant chez les bénéficiaires âgés que chez les aidants familiaux selon lequel l'entretien ménager est un service nécessaire, voire indispensable 
dans le contexte des services de maintien à domicile que l'État doit offrir, celui-ci choisit par un étrange paradoxe de le retirer de sa liste (MSSS, 1990a). Paradoxe, car au moment où l'État désire se rapprocher du citoyen - d'où le titre Une réforme axée sur le citoyen - il retranche I'un des services les plus sollicités et appréciés par celui-ci.

Partenariat ou soliloque institutionnalisé entre les familles et I'État? L'examen n'est pas définitif. Une première génération de recherches a posé les jalons d'une réflexion préliminaire. De larges pans demeurent encore occultés (par exemple, le partage souhaitable entre I'État et l'aide naturelle sur le plan des tâches et des responsabilités en maintien à domicile, une connaissance plus systématique des typologies familiales, des systèmes de valeurs oscillant entre autonomie et dépendance...). La réflexion est encore jeune.

Avant d'aborder le dernier stade de notre itinéraire sur le maintien à domicile, il nous faut mentionner la présence $d^{\prime}$ un autre acteur: le privé. De fait, depuis le début de la décennie 80 en particulier, on a assisté à une prolifération croissante de la présence du secteur privé, tant dans le maintien à domicile (par l'entremise des services d'aide à domicile, mais aussi de soins d'hygiène et infirmiers) que dans celui de I'hébergement institutionnel (multiplication de résidences privées de toutes sortes, illicites ou autorisées, conventionnées ou non). Déjà en 1984-1985, le tiers des CLSC dirigeaient leur clientèle vers des banques de ressources privées pour obtenir des services d'aide à domicile (Roy, 1986). Quatre ans plus tard (1988-1989), les deux tiers des CLSC y recouraient (MSSS, 1990b). Maintenant, les documents ministériels autorisent (sinon encouragent) cette voie vers la privatisation (Pelletier et al., 1991; MSSS, 1990a). Celle-ci quitte progressivement le vestiaire de l'anonymat pour s'affirmer au grand jour. Par ailleurs, l'impact de ce courant de privatisation dans le secteur du maintien à domicile $\mathrm{n}^{\prime} \mathrm{a}$ pas encore été évalué d'une façon globale. Ce que l'on sait par ailleurs, $c^{\prime}$ est que le mouvement semble irréversible.

\section{QUATRIÈME STADE: Un maintien à domicile qui tourne en rond}

Nous en sommes à l'époque actuelle. Une époque porteuse de son passé récent - des clientèles toujours plus lourdes, des familles et des communautés davantage mises à contribution. Certains estimeront que c'est la loi de Murphy qui prévaudra dans l'avenir - ce qui est pire empire -; d'autres auront foi en de nouveaux modèles de pratique, à l'avènement d'une nouvelle génération de personnes âgées moins dépendantes des services. Les deux versions peuvent même coexister en partie. 
Une époque garante de son passé, oui, mais qui tourne en rond, qui fait du surplace dans un environnement qui, par ailleurs, évolue d'une façon fulgurante, où les clientèles ne cessent de s'adresser de toutes parts aux portes des services de maintien à domicile. Cet « environnement » est créé par des facteurs ou phénomènes sociaux extérieurs au maintien à domicile, mais qui le conditionnent d'une façon décisive. Le vieillissement accéléré de la population, la diminution du soutien communautaire dans les communautés en voie de désintégration démographique et sociale, les mutations familiales, la participation accrue des femmes au marché du travail, la mobilité géographique et professionnelle des enfants, l'individualisme montant, la saturation des ressources institutionnelles, l'érosion de l'universalité et de la gratuité des services, etc., sont autant de déterminants qui exercent une pression constante sur le maintien à domicile, en font un «champ éclaté » où les points de repère sont trop nombreux pour prédire les clientèles à venir.

Trois dimensions tenant au discours officiel, aux actions gouvernementales et aux pratiques en CLSC nous font considérer que le maintien à domicile tourne en rond, sans connaître l'évolution désirée. En premier lieu, le discours officiel: il est étonnant de constater que la dernière politique de services de maintien à domicile date de 1979 . Pourtant, depuis cette date, le réseau des affaires sociales s'est métamorphosé considérablement et l'État a toujours signifié sa volonté d'orienter ses services vers des ressources légères et en milieu naturel (MAS, 1985; MSSS, 1989a, 1990a et 1992a). Entre autres, trois documents du Ministère (MSSS, 1991, 1993 et 1994) faisaient état de l'objectif de réduire à $5 \%$ le taux d'hébergement institutionnel au Québec. Ce qui est considérable comme visée si I'on tient compte qu'à I'heure actuelle, avec $7 \%$ de personnes âgées en institution, le Québec figure encore parmi les pays occidentaux enregistrant les taux les plus élevés d'hébergement institutionnel.

Ce n'est pas que le ministère de la Santé et des Services sociaux $\mathrm{n}$ 'ait pas consenti des efforts à la réflexion et à la rédaction de documents sur le sujet. Au contraire, l'activité «plumitive » du Ministère fut des plus fébriles depuis 1980. Analyses, études, rapports furent produits. Mentionnons principalement l'existence de trois rapports résultant de groupes de travail sur le maintien à domicile (MAS, 1983, 1984 ; MSSS, 1990b). Ces rapports ont fourni une réflexion importante sur les éléments de révision de la politique des services de maintien à domicile, sur la nature des besoins des populations âgées à domicile, enfin sur la problématique de l'allocation directe. Puis, on s'est employé à l'élaboration de textes de politiques (MSSS, 1988, 1991, 1992b et 1993). Des titres tels que Les services à domicile. Cadre de référence, État de situation, Politique de services à domicile pour les personnes qui présentent 
des limitations d'activités et leur milieu respectif font partie du répertoire remisé dans l'armoire. Sans compter la panoplie de comités de travail portant sur la révision (sic) de la politique de maintien à domicile. Un fonctionnaire fêtait tout récemment son onzième anniversaire de participation à de tels comités en la matière.

Malgré l'effervescence de la réflexion, le Ministère ne s'est nullement commis, sauf exception, dans un document de politique orientant le maintien à domicile. Il existe çà et là des mesures administratives spécifiques, des points de réglementation et des règles d'allocation des ressources qui font office de paramètres. Sans plus! Une exception cependant: un document intitulé Les services à domicile de première ligne. Cadre de référence (MSSS, 1994) vient préciser certains paramètres de gestion des services et évoque certains principes de base régissant la complémentarité des ressources socio-sanitaires. Malgré ce progrès visible, plusieurs questions visant le milieu des pratiques n'obtiennent pas de réponse dans ce document (Alors, l'hémodialyse, on l'inclut ou non dans les services? Les soins palliatifs devraient-ils être offerts dans tous les CLSC? Les services intensifs (SIMAD), en principe, devraient-ils être existants dans toutes les régions? C'est quoi les critères définissant une personne socio-économiquement défavorisée aux fins de gestion des services d'aide à domicile? etc.).

La seconde dimension pouvant expliquer que le maintien à domicile «tourne en rond» repose sur le fait que l'action gouvernementale $\mathrm{n}^{\prime}$ appuie pas le discours officiel (existant, mais orphelin d'une politique). Les auteurs du rapport Pelletier sur les personnes âgées (Pelletier et al., 1991) rendent compte de l'équivoque:

La majeure partie de l'investissement se fait dans le secteur institutionnel (hôpitaux, hébergement), ne laissant qu'une faible part des ressources au maintien à domicile. II est remarquable de constater l'écart qui subsiste entre les choix budgétaires et les discours qui prônent une réponse aux besoins et aux désirs des aînés ${ }^{11}$.

Ainsi, en 1989-1990, le maintien à domicile ne représentait que $8,4 \%$ du budget total destiné aux personnes âgées, les ressources d'hébergement accaparant la quasi-totalité du budget (hôpitaux et centres d'accueil et d'hébergement confondus) (Pelletier et al., 1991).

Cette contradiction entre le discours et l'allocation des ressources, on l'a déjà évoquée dans un stade antérieur de l'évolution du maintien à domicile. Ce qui est particulier ici, c'est que la situation ne s'est pas

11. J. Pelletier et al. Vers un nouvel équilibre des âges, Rapport du groupe d'experts sur les personnes aînées, Québec: Gouvernement du Québec, 1991, p. 57. 
réellement corrigée depuis, malgré une décennie de discours annonçant un virage vers le maintien à domicile et certains progrès timides notés ici et là (en particulier, l'ajout de ressources pour les services intensifs SIMAD en 1986). Avec 239 millions de dollars en 1993-1994 en maintien à domicile, l'État québécois a un per capita de dépenses d'à peine la moitié de celui de l'Ontario et du tiers de celui d'un pays comme la Suède, par exemple (MSSS, 1994).

Le document Une réforme axée sur le citoyen (MSSS, 1990a) prévoyait doubler en cinq ans les ressources en maintien à domicile. Cette mesure est demeurée depuis lettre morte, à l'exception d'un léger progrès pour l'année 1994-1995 (12 millions) à partager avec le réseau des centres d'accueil et de soins de longue durée. On est loin des 40 millions par année prévus dans le document sur la réforme. Parallèlement, l'évaluation par la Commission Rochon (1987) selon laquelle il faudrait multiplier par trois ou quatre le budget en maintien à domicile pour répondre aux besoins de la clientèle tient toujours; elle n'a pas disparu pour autant. On imagine alors le casse-tête - sinon l'impasse - sur le front des services.

Quant aux pratiques - c'est notre dernier point-, elles se cherchent. Une récente recherche-consultation qui a eu lieu au printemps 1993 auprès des 39 CLSC des régions du Bas-Saint-Laurent, de la Gaspésieîles-de-la-Madeleine, de Québec et de Trois-Rivières (Roy, Saillant et Vézina, 1994) ${ }^{12}$ révèle les interrogations suivantes: Quelles sont les limites de l'aide naturelle? Que peut-on demander aux familles? Quel est le meilleur partage de responsabilités État-famille? Comment résoudre la contradiction entre des pratiques autonomisantes et des valeurs de dépendance chez certains usagers? L'allocation directe sert-elle les objectifs du maintien à domicile? L'entretien ménager doit-il être conservé dans les services? Existe-t-il des typologies de clientèle pour lesquelles la prestation des services et la façon d'intervenir seraient différentes? Certains cas très lourds ne devraient-ils pas être ailleurs qu'en maintien à domicile? Comment faire le partage entre l'utopie et la réalité dans le discours officiel et les politiques locales de services? Autant de questions qui couvrent d'ombres et d'incertitudes les pratiques. La liste n'est pas exhaustive.

Dans un tel contexte, les intervenants sont les apôtres d'une mission infinie, résultant de l'évolution des politiques sociales de l'État, de

12. Il s'agit d'une consultation effectuée dans le cadre d'une démarche commune de programmation de recherche en soutien à domicile (Centre de recherche sur les services communautaires de I'Université Laval / CLSC des quatre régions). Une subvention du Conseil québécois de la recherche sociale permet la réalisation de ce projet de programmation étalé sur un an et demi. 
la mutation des forces sociales et de la famille ainsi que de l'impasse budgétaire du gouvernement. Toutes les clientèles du réseau frappent à leurs portes. Usagers et intervenants tiennent le langage des contraintes. Parallèlement, une évidence s'impose: la seule vertu du maintien à domicile, sans des moyens pour la soutenir, ne peut à elle seule triompher. On a beau carburer à la foi, dire que le milieu naturel et les ressources de maintien à domicile sont la solution pour tous, la foi tout en déplaçant des montagnes ne pourra guérir les grands malades ni se substituer aux familles absentes ou fragiles, sans moyens.

Dans ce dernier stade, certains avanceront que le maintien à domicile tourne en rond effectivement. D'autres affirmeront qu'au contraire ça ne tourne pas rond chez lui. Ils diront un peu la même chose.

\section{CONCLUSION}

L'article n'épuise nullement l'histoire récente du maintien à domicile. Chacun et chacune pourraient y ajouter un ingrédient, une interprétation ou une nouvelle prise d'angle qui compléterait et enrichirait (ou nuancerait) I'itinéraire du maintien à domicile tel que tracé ici. Tout au plus croyons-nous avoir exposé les éléments de contexte général dans lesquels s'inscrit l'évolution du maintien à domicile au Québec.

Les quatre stades évolutifs contiennent leur part d'arbitraire; on aurait pu imaginer d'autres séquences, d'autres manières de les présenter. Néanmoins, ils peuvent avoir le mérite de guider la réflexion sur les principales étapes décisives du maintien à domicile. Avouons-le, le champ du maintien à domicile, éclaté, traversé par les politiques sociales de l'État, est fascinant à observer pour les chercheurs (peut-être un peu moins euphorisant pour les apôtres-intervenants qui évoluent au front). II est ouvert potentiellement à toutes les clientèles du réseau de la santé et des services sociaux; il est le réceptacle premier des politiques de désinstitutionnalisation; il pose des questions de société (rapports État-familles, privatisation des services, systèmes de valeurs usagers-services, questions d'équité sur le plan des ressources par catégorie de clientèle, par régions socio-sanitaires...); enfin, il est intimement lié à l'avenir, notamment en raison du vieillissement accéléré de la population et du désengagement progressif de l'État, deux phénomènes qui se suivent parallèlement.

Pour l'heure, le maintien à domicile demeure un chantier ouvert à la recherche et à la réflexion. Son histoire s'entremêle avec celle de I'État, de ses politiques sociales, de ses orientations et de ses fréquents 
changements de cap. À travers les incertitudes qui pèsent pour demain, il est une certitude: on ne pourra jamais dissocier le maintien à domicile de l'histoire de tout ce qui l'alimente et le commet. C'est-à-dire à peu près tout.

\section{Références bibliographiques}

BÉLAND, F. (1982). Les principaux résultats de l'analyse des désirs d'hébergement de trois échantillons de personnes âgées du Québec. Québec: ministère des Affaires sociales.

BÉLAND, F. (1983). L'évaluation de la politique des services à domicile du MAS, Étude de cas. Québec: ministère des Affaires sociales.

BÉLAND, F. (1984). "L'effet de la corésidence sur les désirs d'hébergement des personnes âgées », Recherches sociographiques, vol. XXV, $\mathrm{n}^{\circ} 2$ : 267-283.

BÉLAND, F. (1987). "Identifying Profile of Service Requirements in a Noninstitutionalized Elderly Population », Journal of Chronical Disease, vol. 40, $\mathrm{n}^{\circ} 1$ : 51-64.

BOLDUC, M. (1981a). L'aide à domicile aux personnes à moyens physiques restreints par la formule d'allocation financière. Québec: Centre FrançoisCharon.

BOLDUC, M. (1981b). Recherche évaluative portant sur le projet expérimental "Logements accessibles et services de support». Québec: Centre François-Charon.

BOLDUC, M. (1986). La politique et les programmes de services à domicile: constats et réflexions évaluatives. Québec: ministère de la Santé et des Services sociaux.

BOLDUC, M. (1988). Individualized Funding. A Summary of the Quebec Experience. Québec: Assessment - Rehabilitation and Long Term Services, ministère de la Santé et des Sevices sociaux.

BOLDUC, M. et L. GARANT (1990). L'aide par les proches: mythes et réalités. Québec: ministère de la Santé et des Services sociaux.

BRINK, S. (1985). "Housing Elderly People in Canada: Working Towards a Continuum of Housing Choices Appropriate to their Needs», dans Gloria Gutman et Norman Blackie (dir.), Innovations in Housing and Living Arrangements for Seniors. Burnaby (Colombie-Britannique): Université Simon-Fraser, p. 1-23.

BRUNET, J. et al. (1987). Rapport du Comité de réflexion et d'analyse des services dispensés par les CLSC. Québec: Gouvernement du Québec.

Bureau De LA STATISTIQUe DU QuÉBEC (1984). Perspectives démographiques régionales, 1981-2006. Québec: Gouvernement du Québec.

Comité De la SANTÉ Mentale (1987). Pour un partenariat élargi, projet de politique de santé mentale pour le Québec. Québec: Gouvernement du Québec.

COMMISSION D'ENQUÊTE SUR LES SERVICES DE SANTÉ ET LES SERVICES SOCIAUX (1988). Rapport de la Commission d'enquête sur les services de santé et les services sociaux. Québec: Gouvernement du Québec. 
COMMISSION ROCHON (1987). Dossier "personnes âgées", Programme de consultation d'experts. Québec: Commission d'enquête sur les services de santé et les services sociaux.

CONSEIL DE LA FAMILLE (1989). Penser et agir famille. Québec: Gouvernement du Québec.

CONSEIL DES AFFAIRES SOCIALES ET DE LA FAMILLE (1984). Objectif: Santé, Rapport du comité d'étude sur la promotion de la santé. Québec: Gouvernement du Québec.

CONSEIL NATIONAL DU BIEN-ÊTRE SOCIAL (1984). Soixante-cinq ans et plus: rapport du Conseil national du bien-être social sur les revenus des personnes âgées. Ottawa: Ministère des Approvisionnements et Services.

CONSEIL RÉGIONAL DE LA SANTÉ ET DES SERVICES SOCIAUX DU MONTRÉAL MÉTROPOLITAIN (1985). Projet de financement des services de maintien à domicile pour les personnes âgées en perte d'autonomie. Survol et envol, 1981-1985. Montréal.

CONTANDRIOPOUlOS, A.-P., D. LAROUCHE et G. TESSIER (1983). «Les effets d'un programme d'aide à domicile québécois sur le profil d'utilisation des ressources socio-sanitaires : une étude de substitution », Communication présentée dans le cadre de Systed 83. Montréal: Université de Montréal.

CONTANDRIOPOUlOS, A.-P. et al. (1984). Les services d'aide à domicile et I'utilisation des ressources du système de soins, Rapport. Montréal: Université de Montréal.

CORIN, E. et al. (1984). "Entre les services professionnels et les réseaux sociaux: les stratégies d'existence des personnes âgées ", Sociologie et sociétés, vol. XVI, $\mathrm{n}^{\circ} 2$, octobre: 89-104.

FÉDÉRATION DES CLSC DU QUÉBEC (1988). Les services à domicile : une responsabilité publique. Montréal. Mémoire présenté à la ministre de la Santé et des Services sociaux, madame Thérèse Lavoie-Roux.

FILIATRAULT, P. et J. LOCAS (1987). Monographie sur les services de maintien à domicile: tendances et problématiques actuelles. Québec: ministère de la Santé et des Services sociaux.

Gouvernement du CANAdA (1986a). Aging: Shifting the Emphasis. Ottawa: Health and Welfare Canada.

GouvernemENT Du CANADA (1986b). A Framework for the Development of Home Care in Canada. Ottawa: Health and Welfare Canada.

GouvERNEMENT DU CANADA (1990). Rapport sur les soins à domicile / Report on Home Care. Ottawa : Approvisionnements et Services, Santé et Bienêtre social Canada.

GRANDMAISON, A. (1982). "La désinstitutionnalisation en regard des personnes âgées: un phénomène paradoxal », Intervention, $\mathrm{n}^{\circ}$ 64 : 12-20.

GUAY, J. et al. (1985). Document d'initiation aux types d'intervention communautaire. Sainte-Foy: Centre de recherche sur les services communautaires, Université Laval.

GUBERMAN, N. (1987). "Discours de responsabilisation de la «famille» et retrait de l'État-providence », Couples et parents des années quatre-vingt. Québec: Institut québécois de recherche sur la culture, p. 193-208.

Guberman, N. et al. (1987). Amour, bain, comprimé ou l'ABC de la désinstitutionnalisation. Québec: Commission d'enquête sur les services de santé et les services sociaux (Recherche, 23). 
HaJjAR, M. et L. LefebVRe (1988). "Soins et services... à domicile », Nursing Québec, vol. 8, n 6, novembre-décembre: 67-70.

JOUBERT, P. et al. (1991). Évaluation du programme québécois de services intensifs de maintien à domicile (SIMAD). Sainte-Foy: Unité de recherche en santé communautaire, Centre hospitalier de l'Université Laval.

JUTRAS, S. et F. VEILLEUX (1989). Des "partenaires" méconnus: Les aidants des personnes âgées en perte d'autonomie. Montréal : Université de Montréal.

LAMBERT, P. et C. DRABBLE (1985). Projet de financement des services de maintien à domicile pour les personnes âgées en perte d'autonomie. Montréal: Conseil régional de la santé et des services sociaux du Montréal métropolitain.

LeSEMANN, F. et C. Chaume (1989). Familles-providence, la part de l'État. Montréal : Les Éditions Saint-Martin.

LESEMANN, F. et C. MARTIN (1993). Les personnes âgées. Dépendance, soins et solidarités familiales. Comparaisons internationales. Paris : La Documentation française.

MATHEWS, G. (1988a). Le vieillissement démographique et son impact sur la situation des personnes âgées et les services qui leur sont offerts, Rapport final de recherche. Montréal: INRS-Urbanisation.

MATHEWS, G. (1988b). Priorités de la recherche en matière de vieillissement. Montréal : INRS-Urbanisation.

MCKNIGHT, J. (1977). "Le professionnalisme dans les services: un secours abrutissant», Sociologie et sociétés, vol. IX, $\mathrm{n}^{\circ} 1:$ 7-19.

Ministère de La SANTÉ et DES ServiCes SOCIAux (1988). Politique de services à domicile, État de situation, chapitre 1. Québec.

MINISTÈre DE LA SANTÉ ET DES SERVICES SOCIAuX (1989a). Pour améliorer la santé et le bien-être au Québec. Orientations. Québec: Gouvernement du Québec.

Ministère de la Santé et des Services sociaux (1989b). Politique de santé mentale. Québec: Gouvernement du Québec.

MINISTÈRE DE LA SANTÉ ET DES SERVICES SOCIAUX (1990a). Une réforme axée sur le citoyen. Québec: Gouvernement du Québec.

MINISTÈRE DE LA SANTÉ ET DES SERVICES SOCIAUX (1990b). Rapport du groupe de travail interdirectionnel portant sur l'allocation directe en maintien à domicile. Québec: Gouvernement du Québec.

Ministère de la SANTÉ et Des Services sociaux (1991). Politique de services à domicile. Document de travail, version novembre. Québec.

MINISTÈRE DE LA SANTÉ ET DES SERVICES SOCIAUX (1992a). La politique de la santé et du bien-être. Québec: Gouvernement du Québec.

Ministère de LA SANTÉ et Des ServiCes SOCIAux (1992b). Politique de services à domicile pour les personnes qui présentent des limitations d'activités et leur milieu respectif. Document de travail, version mai. Québec.

MINISTÈRE DE LA SANTÉ ET DES SERVICES SOCIAUX (1993). Les services à domicile. Cadre de référence. Document de travail, version avril. Québec.

MINISTÈRE DE LA SANTÉ ET DES SERVICES SOCIAUX (1994). Les services à domicile de première ligne. Cadre de référence. Québec: Gouvernement du Québec.

MINISTÈRE DES AFFAIRES SOCIALES (1979). Les services à domicile: politique du ministère des Affaires sociales. Québec. 
MINISTÈRE DES AFFAIRES SOCIALES (1983). Rapport d'étape du groupe de travail sur la révision de la politique des services à domicile du ministère des Affaires sociales. Québec.

Ministère Des AfFAires SOCIALES (1984). Population âgée à domicile. Nature des besoins et éléments de réponse. Rapport du groupe de travail sur les alternatives à I'institutionnalisation. Québec.

MINISTÈRE DES AFFAIRES SOCIALES (1985). Un nouvel âge à partager: politique du ministère des Affaires sociales à l'égard des personnes âgées. Québec: Gouvernement du Québec.

OFFICE DES PERSONNES HANDICAPÉES DU QuÉBEC (1984). À part égale... L'intégration sociale des personnes handicapées. Québec : Gouvernement du Québec.

PANET-RAYMOND, J. (1987). "Le rapport Barclay et le leurre du "communautaire" », Intervention, $\mathrm{n}^{\circ} 78: 50-57$.

PeLLetIER, J. et al. (1991). Vers un nouvel équilibre des âges. Rapport du groupe d'experts sur les personnes aînées. Québec: Gouvernement du Québec.

ROBERGE, H. (1987). "Le programme de maintien à domicile, "baril sans fond" ou "baril de poudre" », La Presse, 4 octobre.

RoY, J. (1986). Bilan du maintien à domicile dans les CLSC, Tome I- Problématique des services, Tome II- Problématique des ressources. Montréal : Fédération des CLSC du Québec.

RoY, J. (1987). "Communauté, désinstitutionnalisation et discours officiel », Service social, vol. $36, \mathrm{n}^{\text {os }} 2$ et $3: 197-206$.

RoY, J. (1988). Projet de politique en maintien à domicile, région de l'Estrie. Sainte-Foy: Centre de recherche sur les services communautaires, Université Laval.

RoY, J. (1989). Étude exploratoire de la situation des personnes âgées vivant à domicile, dans le secteur Des Chenaux, et leur désir d'hébergement institutionnel. Sainte-Foy: Centre de recherche sur les services communautaires, Université Laval.

RoY, J. (1990a). «Familles et politiques sociales: la question du partenariat avec la famille sur le plan des politiques de services», dans État et familles : des politiques sociales en mutation. Québec: Collection Études et recherches, Conseil de la famille du Québec, p. 11-21.

ROY, J. (1990b). «Les personnes âgées vivant à domicile et le désir d'hébergement institutionnel », Recherches sociographiques, vol. XXXI, n $2: 227-239$.

ROY, J., A. VÉZINA et M. PARADIS (1992). De la tâche à la mission. Personnes âgées, aidants naturels, services intensifs de maintien à domicile (SIMAD). Sainte-Foy: Centre de recherche sur les services communautaires, Université Laval.

ROY, J., F. SAILLANT et A. VÉZINA (1994). Priorités de recherche en soutien à domicile. Bilan des consultations en CLSC. Sainte-Foy: Centre de recherche sur les services communautaires, Université Laval.

SicOTTE, C. (1982). Évaluation des bénéficiaires en centres d'accueil d'hébergement et en centres hospitaliers de soins prolongés. Québec: ministère des Affaires sociales.

THERRIEN, R. (1989). "La responsabilité des familles et des femmes dans le maintien à domicile des personnes âgées : une politique de désengagement ou de soutien de l'État », Santé mentale au Québec, vol. XIV, n 1 : 152-164. 
TRAHAN, L. (1989). Les facteurs associés à l'orientation des personnes âgées dans des établissements d'hébergement: une revue de la littérature. Québec: Ministère de la santé et des services sociaux.

TRÉPANIER, A. (1985). Perspectives d'avenir à propos du projet " cas lourds». Montréal : Conseil régional de la santé et des services sociaux du Montréal métropolitain.

TRÉPANIER, A. (1986). Services à domicile dans la région 06A : éléments du système de santé et des services sociaux à développer en priorité. Montréal: Conseil régional de la santé et des services sociaux du Montréal métropolitain.

TRÉPANIER, A. et P. LAMBERT (1987). Programme de services intensifs à domicile: Rapport d'activités et bilan statistique 1986-87. Montréal : Conseil régional de la santé et des services sociaux du Montréal métropolitain.

ZAY, N. (1984). "Analyse critique des politiques et des institutions québécoises concernant les personnes âgées », Sociologie et sociétés, vol. XVI, $n^{\circ} 2$ : 105-118. 